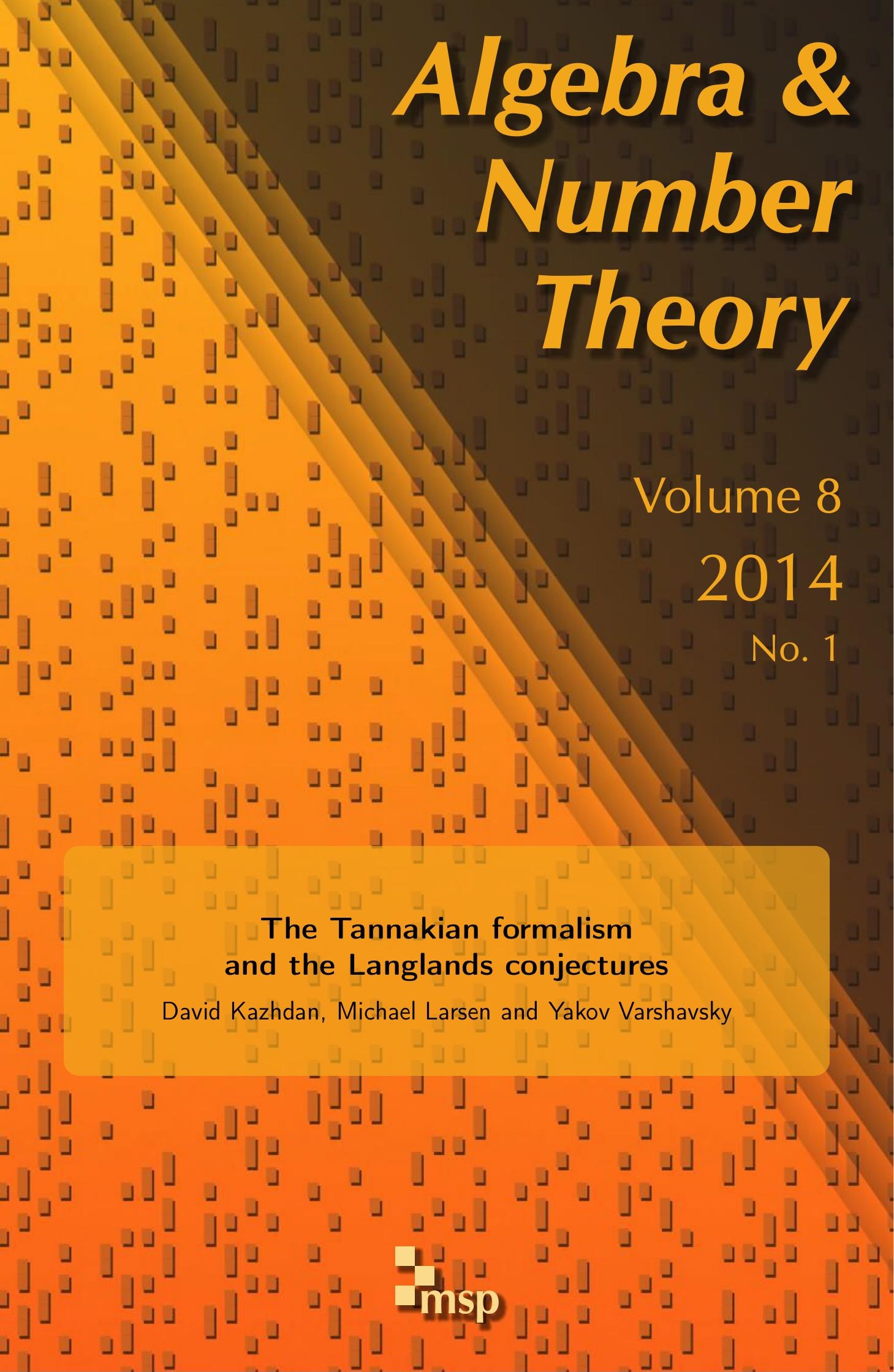




\title{
The Tannakian formalism and the Langlands conjectures
}

\author{
David Kazhdan, Michael Larsen and Yakov Varshavsky
}

Let $H$ be a connected reductive group over an algebraically closed field of characteristic zero, and let $\Gamma$ be an abstract group. In this note, we show that every homomorphism of Grothendieck semirings $\phi: K_{0}^{+}[H] \rightarrow K_{0}^{+}[\Gamma]$, which maps irreducible representations to irreducible, comes from a group homomorphism $\rho: \Gamma \rightarrow H(K)$. We also connect this result with the Langlands conjectures.

\section{Introduction}

Let $F$ be a global function field, $\Gamma_{F}$ the absolute Galois group of $F, G$ a split connected reductive group over $F, \ell$ a prime number different from the characteristic of $F$, and $\hat{G}={ }^{L} G^{0}$ the connected Langlands dual group over $\overline{\mathbb{Q}}_{\ell}$.

Recall that a weak Langlands conjecture asserts that for every pair $(\pi, \omega)$, where $\pi$ is an automorphic representation of $G$, whose central character is of finite order, and $\omega$ is a representation of $\hat{G}$, there exists a unique semisimple $\ell$-adic representation $\rho_{\pi, \omega}$ of $\Gamma_{F}$, whose $L^{S}$-function is equal to the $L^{S}$-function of $(\pi, \omega)$.

Moreover, a strong Langlands conjecture asserts that there exists a $\hat{G}$-valued $\ell$-adic representation $\rho_{\pi}: \Gamma_{F} \rightarrow \hat{G}\left(\overline{\mathbb{Q}}_{\ell}\right)$ (not unique in general) such that the composition $\omega \circ \rho_{\pi}$ is isomorphic to $\rho_{\pi, \omega}$ for each representation $\omega$.

The main result of this note implies that in some cases the strong Langlands conjecture follows from the weak one. More specifically, we show the existence of $\rho_{\pi}$ in the case when $\rho_{\pi, \omega}$ is irreducible for each irreducible representation $\omega$. In this case, $\rho_{\pi}$ is unique up to conjugation, and the Zariski closure of its image contains the derived group of $G$.

Our result is a corollary of the following variant of the Tannakian formalism. Let $H$ be a connected reductive group over an algebraically closed field $K$ of characteristic zero, and let $\Gamma$ be an abstract group. Then every homomorphism of groups $\rho: \Gamma \rightarrow H(K)$ induces a homomorphism of Grothendieck semirings

Kazhdan was partially supported by ISF grant 1438/06. Larsen was partially supported by NSF grant DMS-0800705. Varshavsky was partially supported by ISF grant 598/09.

MSC2010: primary 11R39; secondary 11F80, 17B10, 18D10.

Keywords: Tannaka duality, Langlands conjectures. 
$\rho^{*}: K_{0}^{+}[H] \rightarrow K_{0}^{+}[\Gamma]$. In this note, we show a partial converse of this assertion. Namely, we show that every homomorphism of Grothendieck semirings $\phi: K_{0}^{+}[H] \rightarrow K_{0}^{+}[\Gamma]$, which maps irreducible representations to irreducibles, comes from a group homomorphism $\rho: \Gamma \rightarrow H(K)$. In particular, we show that a connected reductive group is determined by its Grothendieck semiring.

This note was inspired by a combination of a work in progress [Kazhdan and Varshavsky $\geq 2014$ ], where it is shown that the weak Langlands conjecture holds in some cases, and a work [Larsen and Pink 1990], which indicates that one does not need the full Tannakian structure in order to reconstruct a connected reductive group.

\section{Main results}

Let $K$ be an algebraically closed field of characteristic zero.

1.1. (a) For every algebraic group $G$ over $K$, we denote by $K_{0}^{+}[G]$ the Grothendieck semiring of the category of rational representations of $G$.

In other words, $K_{0}^{+}[G]$ is the set of equivalence classes of finite dimensional semisimple representations of $G$. For every representation $\omega$ of $G$, we denote by $[\omega]$ its class (or more precisely, the class of its semisimplification) in $K_{0}^{+}[G]$. For every pair of semisimple representations $\omega_{1}$ and $\omega_{2}$ of $G$, we have $\left[\omega_{1}\right]+\left[\omega_{2}\right]=\left[\omega_{1} \oplus \omega_{2}\right]$ and $\left[\omega_{1}\right] \cdot\left[\omega_{2}\right]=\left[\omega_{1} \otimes \omega_{2}\right]$.

(b) Note that a representation $\omega$ of $G$ is irreducible if and only if its class $[\omega] \in$ $K_{0}^{+}[G]$ is irreducible, that is, it cannot be realized as a nontrivial sum $\left[\omega_{1}\right]+\left[\omega_{2}\right]$ of elements of $K_{0}^{+}[G]$.

(c) Every homomorphism $\rho: G \rightarrow H$ of algebraic groups over $K$ gives rise to the homomorphism $\rho^{*}: K_{0}^{+}[H] \rightarrow K_{0}^{+}[G]$ of semirings, where $\rho^{*}([\omega]):=[\omega \circ \rho]$.

The following result asserts that each connected reductive group is determined by its Grothendieck semiring:

Theorem 1.2. Let $G$ and $H$ be two connected reductive groups over $K$, and let $\phi: K_{0}^{+}[G] \stackrel{\sim}{\longrightarrow} K_{0}^{+}[H]$ be an isomorphism of semirings.

Then there exists an isomorphism $\rho: H \stackrel{\sim}{\longrightarrow} G$ such that $\rho^{*}=\phi$. Moreover, $\rho$ is unique up to conjugation.

Remark 1.3. Note, by comparison, that, if $G$ is connected, semisimple, and simply connected, then the Grothendieck ring $K_{0}[G]$ is isomorphic to $\mathbb{Z}\left[x_{1}, \ldots, x_{r}\right]$, where $r$ is the rank of $G$. Thus, for such groups, $K_{0}[G]$ encodes only the rank.

1.4. Now let $\Gamma$ be an abstract group, and let $K_{0}^{+}[\Gamma]$ be the Grothendieck semiring of the category of finite-dimensional representations of $\Gamma$ over $K$. Every group homomorphism $\rho: \Gamma \rightarrow G(K)$ gives rise to the homomorphism $\rho^{*}: K_{0}^{+}[G] \rightarrow$ $K_{0}^{+}[\Gamma]$ of Grothendieck semirings. 
We have the following version of the Tannakian formalism:

Theorem 1.5. Let $\phi: K_{0}^{+}[G] \rightarrow K_{0}^{+}[\Gamma]$ be a homomorphism of semirings that maps irreducible elements to irreducibles.

Then there exists a homomorphism $\rho: \Gamma \rightarrow G(K)$ such that $\rho^{*}=\phi$. Moreover, the Zariski closure of the image of each such $\rho$ contains $G^{\mathrm{der}}$, and $\rho$ is unique up to conjugation.

Remarks 1.6. (a) Conversely, let $\rho: \Gamma \rightarrow G(K)$ be a homomorphism such that the Zariski closure of $\rho(\Gamma)$ contains $G^{\text {der }}$. Then the homomorphism $\rho^{*}: K_{0}^{+}[G] \rightarrow$ $K_{0}^{+}[\Gamma]$ maps irreducible elements to irreducibles.

(b) The result fails completely if one does not assume that $\phi$ maps irreducible elements to irreducible.

Indeed, let $G$ be $\mathrm{SL}_{2}$, and let $\Gamma$ be the group with one element. In this case, for each integer $k \geq 2$, there exists a (unique) homomorphism of semirings

$$
\phi_{k}: K_{0}^{+}\left[\mathrm{SL}_{2}\right] \rightarrow K_{0}^{+}[\Gamma]=\mathbb{Z}_{\geq 0},
$$

which maps the standard representation of $\mathrm{SL}_{2}$ to $k \in \mathbb{Z}_{\geq 0}$. Only $\phi_{2}$ corresponds to a (unique) homomorphism $\Gamma \rightarrow \mathrm{SL}_{2}(K)$.

1.7 (Chevalley space). (a) Let $c_{G}:=\operatorname{Spec} K[G]^{G}$ be the Chevalley space of $G$, where the action of $G$ on $K[G]$ is induced by the adjoint action of $G$ on itself. For every representation $\omega$ of $G$, its trace $\operatorname{Tr}_{\omega} \in K[G]^{G} \subset K[G]$ is a regular function on $c_{G}$.

(b) Let $\chi_{G}: G \rightarrow c_{G}$ be the canonical projection, induced by the embedding $K\left[c_{G}\right]=K[G]^{G} \hookrightarrow K[G]$. Then for each $g \in G$ and each representation $\omega$ of $G$, we have an equality $\operatorname{Tr}_{\omega}\left(\chi_{G}(g)\right)=\operatorname{Tr}_{\omega}(g)$.

The following result is a more explicit formulation of Theorem 1.5:

Corollary 1.8. Let $f: \Gamma \rightarrow c_{G}(K)$ be a map of sets.

Suppose that, for every irreducible algebraic representation $\omega$ of $G$, there exists an irreducible finite-dimensional representation $\rho_{\omega}$ of $\Gamma$ over $K$ such that

$$
\operatorname{Tr}_{\rho_{\omega}}(\gamma)=\operatorname{Tr}_{\omega}(f(\gamma)) \quad \text { for all } \gamma \in \Gamma .
$$

Then there exists a homomorphism $\rho: \Gamma \rightarrow G(K)$ such that

$$
\chi_{G}(\rho(\gamma))=f(\gamma) \quad \text { for all } \gamma \in \Gamma .
$$

The Zariski closure of $\rho(\Gamma)$ contains $G^{\mathrm{der}}$, and $\rho$ is unique up to conjugation.

Remark 1.9. Conversely, assume that there exists a homomorphism $\rho: \Gamma \rightarrow G(K)$ satisfying (1-2) and such that the Zariski closure of $\rho(\Gamma)$ contains $G^{\text {der }}$. Then for 
every irreducible representation $\omega: G \rightarrow \mathrm{GL}_{n}$ the representation

$$
\rho_{\omega}:=\omega \circ \rho: \Gamma \rightarrow \operatorname{GL}_{n}(K)
$$

is irreducible and satisfies (1-1) (use 1.7(b)).

1.10 (Application to the Langlands conjectures). Let $F$ be a global field, $F^{\text {sep }}$ a separable closure, $\Gamma_{F}=\operatorname{Gal}\left(F^{\mathrm{sep}} / F\right)$ the absolute Galois group, and $\ell$ a prime number different from the characteristic of $F$. Let $\hat{G}$ be a connected reductive group over $\overline{\mathbb{Q}}_{\ell}$.

By $\ell$-adic and $\hat{G}$-valued $\ell$-adic representations of $\Gamma_{F}$, we mean continuous homomorphisms $\rho: \Gamma_{F} \rightarrow \mathrm{GL}_{n}\left(\overline{\mathbb{Q}}_{\ell}\right)$ and $\rho: \Gamma_{F} \rightarrow \hat{G}\left(\overline{\mathbb{Q}}_{\ell}\right)$, respectively, which are unramified for almost all places of $F$.

There are well-defined traces $\operatorname{Tr}_{\rho}\left(\operatorname{Frob}_{v}\right)$ and $\operatorname{Tr}_{\omega \circ \rho}\left(\operatorname{Frob}_{v}\right)$ for almost all places $v$ of $F$ and all representations $\omega$ of $\hat{G}$, respectively.

The following analogue of Corollary 1.8 has applications to Langlands conjectures:

Corollary 1.11. Let $\hat{G}$ be a reductive group over $\overline{\mathbb{Q}}_{\ell}, \Sigma$ a cofinite subset of the set of places of $F$, and $f: \Sigma \rightarrow c_{\hat{G}}\left(\overline{\mathbb{Q}}_{\ell}\right)$ any map of sets.

Assume that, for every irreducible algebraic representation $\omega$ of $G$, there exists an irreducible $\ell$-adic representation $\rho_{\omega}$ of $\Gamma_{F}$ such that

$$
\operatorname{Tr}_{\rho_{\omega}}\left(\operatorname{Frob}_{v}\right)=\operatorname{Tr}_{\omega}(f(v)) \quad \text { for almost all } v \in \Sigma .
$$

Then there exists a $\hat{G}$-valued $\ell$-adic representation $\rho: \Gamma_{K} \rightarrow \hat{G}\left(\overline{\mathbb{Q}}_{\ell}\right)$ such that

$$
\chi_{\hat{G}}\left(\rho\left(\operatorname{Frob}_{v}\right)\right)=f(v) \text { for almost all } v \in \Sigma .
$$

The Zariski closure of $\rho\left(\Gamma_{F}\right)$ contains $\hat{G}^{\mathrm{der}}$, and $\rho$ is unique up to conjugation.

\section{Determining a connected reductive group from its Grothendieck semiring}

In this section, we are going to prove Theorem 1.2. Michael Mueger called our attention to the fact that at least two proofs of this theorem already exist in the literature: [McMullen 1984] and [Handelman 1993]. Nevertheless, we feel that this new proof has merits (including brevity) that justify presenting it.

Let $G$ be a connected reductive group. We will fix a Borel subgroup $B \subset G$ and a maximal torus $T \subset B$. Let $\alpha_{1}, \ldots, \alpha_{r}$ be the simple roots of $G$ with respect to $(B, T)$, and let $W$ be the Weyl group of $(G, T)$.

2.1. (a) We set $U:=X^{*}(T) \otimes \mathbb{R}$. For each subset $X \subset U$, we denote by $\operatorname{Conv}(X) \subset U$ the convex hull of $X$.

(b) For each dominant weight $v$ of $G$, we denote by $V_{v}$ the irreducible representation of $G$ with highest weight $\nu$. 
(c) We define a partial order on $X^{*}(T)$ by the rule

$$
\mu \leq \lambda \quad \text { if and only if } \quad \lambda=\mu+\sum_{i=1}^{r} x_{i} \alpha_{i} \text { and } x_{i} \geq 0 \text { for all } i
$$

Proposition 2.2. Let $\mu$ and $\lambda$ be two dominant weights of $G$. The following conditions are equivalent:

(a) $\mu \leq \lambda$.

(b) $\operatorname{Conv}(W \mu) \subset \operatorname{Conv}(W \lambda)$.

(c) There exists a finite-dimensional representation $V^{\prime}$ of $G$ such that, for every $n$, every irreducible factor of $V_{\mu}^{\otimes n}$ is a factor of $V_{\lambda}^{\otimes n} \otimes V^{\prime}$.

Proof. (a) $\Longrightarrow$ (b). Notice that, since $\mu$ is dominant, we have $w \mu \leq \mu$ for all $w \in W$. Therefore, our assumption $\mu \leq \lambda$ implies that $w \mu \leq \lambda$ for all $w \in W$. Thus, our assertion follows from the following lemma:

Lemma 2.3. Let $\mu$ and $\lambda$ be two weights of $G$ such that $w \mu \leq \lambda$ for all $w \in W$. Then $\operatorname{Conv}(W \mu) \subset \operatorname{Conv}(W \lambda)$.

Proof. Suppose $\operatorname{Conv}(W \mu)$ is not contained in $\operatorname{Conv}(W \lambda)$. Then there exists $w \in W$ such that $w \mu \notin \operatorname{Conv}(W \lambda)$. As $\operatorname{Conv}(W \lambda)$ is $W$-stable, it follows that $\mu \notin \operatorname{Conv}(W \lambda)$ and hence also $w \mu \notin \operatorname{Conv}(W \lambda)$ for all $w \in W$.

By the separation lemma, there exists $\theta \in U^{*}$ such that $\theta(\mu)>\theta(w \lambda)$ for all $w \in W$. This is an open condition, so we may choose $\theta$ such that $\theta\left(\alpha_{i}\right) \neq 0$ for each $i=1, \ldots, r$. Replacing $\theta$ by $w \theta$ and $\mu$ by $w \mu$ for some $w \in W$, we may assume in addition that $\theta\left(\alpha_{i}\right)>0$ for each $i=1, \ldots, r$.

By our assumption, $\mu=\lambda-\sum_{i=1}^{r} x_{i} \alpha_{i}$ with each $x_{i} \geq 0$. Therefore,

$$
\theta(\mu)=\theta(\lambda)-\sum_{i=1}^{r} x_{i} \theta\left(\alpha_{i}\right) \leq \theta(\lambda)
$$

contradicting our assumption $\theta(\mu)>\theta(\lambda)$.

(b) $\Longrightarrow$ (c). We start with the following lemma:

Lemma 2.4. Let $X$ be a finite subset of a finite-dimensional Euclidean space $E$. Then there exists a compact subset $Y$ of $E$ such that

$$
\operatorname{Conv}(n X) \subset Y+\underbrace{X+X+\cdots+X}_{n}
$$

for all positive integers $n$. 
Proof. Let $m:=|X|$, and let $Y$ denote the ball of vectors of norm at most $R:=$ $2 m \max _{x \in X}\|x\|$. We claim that inclusion (2-1) holds for this $Y$.

Let $X$ be the set $\left\{x_{1}, \ldots, x_{m}\right\}$. Then every vector in $\operatorname{Conv}(n X)$ is of the form

$$
v:=a_{1} n x_{1}+\cdots+a_{m} n x_{m},
$$

where the $a_{i}$ are nonnegative and sum to 1 . Let $b_{i}:=\left\lfloor n a_{i}\right\rfloor$ for $i \geq 2$ and $b_{1}=$ $n-\left(b_{2}+\cdots+b_{m}\right)$. As $\left|b_{i}-a_{i} n\right|<1$ for $i>1$, we have

$$
\left|b_{1}-a_{1} n\right|=\left|n-\left(b_{2}+\cdots+b_{m}\right)+\left(a_{2} n+\cdots+a_{m} n-n\right)\right|<m-1 .
$$

Thus,

$$
\left\|\left(b_{1} x_{1}+\cdots+b_{m} x_{m}\right)-v\right\| \leq \sum_{i=1}^{m}\left|b_{i}-a_{i} n\right|\left\|x_{i}\right\|<R,
$$

and of course, $b_{1} x_{1}+\cdots+b_{m} x_{m}$ belongs to the $n$-fold iterated sum of $X$.

Now we return to the proof of the proposition. We assume that $\operatorname{Conv}(W \mu) \subset$ $\operatorname{Conv}(W \lambda)$, let $X=W \lambda$, and fix a compact set $Y$ satisfying (2-1). Denote by $V^{\prime}$ the direct sum of all representations $V_{v}$ where $v$ ranges over the dominant weights in $W Y$.

If $n$ is a positive integer, the highest weight $\chi$ of any irreducible factor of $V_{\mu}^{\otimes n}$ is a weight of $V_{\mu}^{\otimes n}$. Therefore, $\chi \leq n \mu$; hence, by the implication (a) $\Longrightarrow$ (b) shown above, $\chi$ is an element of

$$
\operatorname{Conv}(W n \mu)=n \operatorname{Conv}(W \mu) \subset n \operatorname{Conv}(W \lambda) .
$$

By (2-1), $\chi$ can be written as a sum of $n$ elements of $W \lambda$ and an element of $W Y$, which is necessarily in the weight group. Thus, $\chi$ has the form $\sum_{i=1}^{n} w_{i} \lambda+w^{\prime} v$ for some $w_{1}, \ldots, w_{n}, w^{\prime} \in W$ and some highest weight $v$ of $V^{\prime}$.

Using the conjecture of Parthasarathy, Ranga Rao, and Varadarajan, proven in [Kumar 1988], we conclude that $V_{\chi}$ is an irreducible factor of $V_{\lambda}^{\otimes n} \otimes V_{\nu}$ and hence also an irreducible factor of $V_{\lambda}^{\otimes n} \otimes V^{\prime}$.

(c) $\Longrightarrow$ (a). Now suppose that there exists a finite-dimensional representation $V^{\prime}$ of $G$ such that, for every $n$, every irreducible factor of $V_{\mu}^{\otimes n}$ must be a factor of $V_{\lambda}^{\otimes n} \otimes V^{\prime}$ as well. Then every weight of $V_{\mu}^{\otimes n}$ must be a weight of $V_{\lambda}^{\otimes n} \otimes V^{\prime}$, and in particular, this is true for the weight $n \mu$. Thus, $n \mu=\lambda_{n}+v_{n}$ for some weights $\lambda_{n}$ of $V_{\lambda}^{\otimes n}$ and $v_{n} \in V^{\prime}$.

Note that $\lambda_{n}=n \lambda-\sum_{i=1}^{r} n_{i} \alpha_{i}$ for some $n_{i} \in \mathbb{Z}_{\geq 0}$. Therefore, $n \mu$ is equal to $n \lambda-\sum_{i=1}^{r} n_{i} \alpha_{i}+v_{n}$; hence, for each $n \in \mathbb{N}$, we have an equality

$$
\lambda-\mu=\sum_{i=1}^{r} \frac{n_{i}}{n} \alpha_{i}-\frac{1}{n} v_{n} .
$$


Next we recall that the set of weights of $V^{\prime}$ is finite, so the expression $(1 / n) v_{n} \in U$ tends to zero when $n$ tends to infinity. Hence, the difference $\lambda-\mu$ equals $\sum_{i=1}^{r} x_{i} \alpha_{i}$, where each $x_{i}=\lim _{n \rightarrow \infty} n_{i} / n$ is nonnegative. This shows that $\mu \leq \lambda$.

Corollary 2.5. The root datum of $G$ can be reconstructed from the semiring $K_{0}^{+}[G]$.

Proof. We divide our construction into steps as follows.

Step 1. First we claim that the partially ordered set of dominant weights of $G$ can be reconstructed from the semiring $K_{0}^{+}[G]$.

For this, we note that the map $\mu \mapsto\left[V_{\mu}\right]$ gives a bijection between the set of dominant weights of $G$ and the set of irreducible objects of $K_{0}^{+}[G]$.

Proposition 2.2 implies that for two dominant weights $\mu$ and $\lambda$ of $G$ we have $\mu \leq \lambda$ if and only if there exists $\theta \in K_{0}^{+}[G]$ such that, for all $n \in \mathbb{N}$ and all irreducible elements $\left[V_{v}\right] \in K_{0}^{+}[G]$, we have

$$
\left[V_{\mu}\right]^{n}-\left[V_{\nu}\right] \in K_{0}^{+}[G] \Longrightarrow\left[V_{\lambda}\right]^{n} \theta-\left[V_{\nu}\right] \in K_{0}^{+}[G] .
$$

Step 2. For every triple $\lambda, \mu, v$ of dominant weights of $G$, we have $\lambda=\mu+v$ if and only if $\lambda$ is the largest dominant weight such that $V_{\lambda}$ is an irreducible factor of $V_{\mu} \otimes V_{\nu}$. Therefore, Proposition 2.2 implies that the semigroup structure on the set of dominant weights of $G$ can be reconstructed from the semiring $K_{0}^{+}[G]$.

Step 3. The group of weights $X^{*}(T)$ of $G$ is the group completion of the semigroup of dominant weights. The group of coweights of $G, X_{*}(T)$, is given as the group of homomorphisms

$$
X_{*}(T)=\operatorname{Hom}\left(X^{*}(T), \mathbb{Z}\right) .
$$

Note that there is a canonical isomorphism between $\operatorname{Aut}\left(X^{*}(T)\right)$ and $\operatorname{Aut}\left(X_{*}(T)\right)$.

Step 4. We claim that $\alpha \in X^{*}(T)$ is a simple root if and only if it is a minimal nonzero weight of $T$ for which there exists a dominant weight $\lambda \in X^{*}(T)$ such that $V_{2 \lambda-\alpha}$ is an irreducible factor of $V_{\lambda}^{\otimes 2}$.

More precisely, we claim that, for every dominant weight $\lambda$, the maximal weights $\mu \neq 2 \lambda$ such that $V_{\mu}$ is an irreducible factor of $V_{\lambda}^{\otimes 2}$ are precisely weights of the form $2 \lambda-\alpha$, where $\alpha$ is a simple root satisfying $\langle\check{\alpha}, \lambda\rangle>0$.

To show this, we observe that every maximal weight $\mu \neq 2 \lambda$ in $V_{\lambda}^{\otimes 2}$ is of the form $2 \lambda-\alpha$, where $\alpha$ is a simple root satisfying $\langle\check{\alpha}, \lambda\rangle>0$. Now the assertion follows from the fact that, for such an $\alpha$, the weight $2 \lambda-\alpha$ has multiplicity one in $V_{2 \lambda}$ and multiplicity two in $V_{\lambda}^{\otimes 2}$.

By Step 1, the set of simple roots can therefore be reconstructed from the semiring $K_{0}^{+}[G]$.

Step 5. For each simple root $\alpha$ of $G$, the corresponding simple coroot $\check{\alpha} \in X_{*}$ can be characterized by the following condition: for every dominant weight $\mu$, the 
pairing $\langle\check{\alpha}, \mu\rangle$ is the unique element $m \in \mathbb{Z}_{\geq 0}$ such that $2 \mu-m \alpha$ is dominant but $2 \mu-(m+1) \alpha$ is not dominant. Indeed,

$$
\langle\check{\alpha}, 2 \mu-m \alpha\rangle=2\langle\check{\alpha}, \mu\rangle-m\langle\check{\alpha}, \alpha\rangle=2\langle\check{\alpha}, \mu\rangle-2 m
$$

is nonnegative if and only if $m \leq\langle\check{\alpha}, \mu\rangle$ while, for every other simple root $\alpha^{\prime} \neq \alpha$ of $G$ with a corresponding simple coroot $\check{\alpha}^{\prime}$, we have

$$
\left\langle\check{\alpha}^{\prime}, 2 \mu-m \alpha\right\rangle=2\left\langle\check{\alpha}^{\prime}, \mu\right\rangle-m\left\langle\check{\alpha}^{\prime}, \alpha\right\rangle \geq 2\left\langle\check{\alpha}^{\prime}, \mu\right\rangle \geq 0
$$

for all $m \geq 0$. Thus, the set of simple coroots can also be reconstructed from $K_{0}^{+}[G]$.

Step 6. After having reconstructed all simple coroots $\check{\alpha}$, we reconstruct all simple reflections $s_{\alpha} \in \operatorname{Aut}\left(X_{*}(T)\right)$, hence the Weyl group $W \subset \operatorname{Aut}\left(X_{*}(T)\right)$, as the subgroup generated by simple reflections. Next we reconstruct the set of all roots of $G$, as images of the simple roots under $W$, and likewise for the coroots of $G$. This completes the reconstruction of the whole root datum of $G$.

2.6. Proof of Theorem 1.2. An isomorphism of semirings $\phi: K_{0}^{+}[G] \stackrel{\sim}{\longrightarrow} K_{0}^{+}[H]$ induces a bijection between irreducible objects and hence a bijection between dominant weights of $G$ and $H$, which we denote by $\tilde{\phi}$.

The proof of Corollary 2.5 shows that $\tilde{\phi}$ extends to an isomorphism between the root data of $G$ and $H$. It therefore comes from an isomorphism of algebraic groups $\rho: H \stackrel{\sim}{\longrightarrow} G$.

We claim that $\rho^{*}: K_{0}^{+}[G] \stackrel{\sim}{\longrightarrow} K_{0}^{+}[H]$ is equal to $\phi$. It is enough to show that, for each dominant weight $\lambda$ of $G$, we have $\phi\left(\left[V_{\lambda}\right]\right)=\rho^{*}\left(\left[V_{\lambda}\right]\right)$. Both expressions, however, are equal to $\left[V_{\tilde{\phi}(\lambda)}\right]$.

Conversely, if $\rho: H \rightarrow G$ is an isomorphism such that $\rho^{*}=\phi$, then for each dominant weight $\lambda$ of $G$ we have $\rho^{*}\left(\left[V_{\lambda}\right]\right)=\phi\left(\left[V_{\lambda}\right]\right)=\left[V_{\tilde{\phi}(\lambda)}\right]$, so $\rho$ induces the isomorphism $\tilde{\phi}$ between the root data; hence, $\rho$ is unique up to conjugation.

\section{The Tannakian formalism}

In this section, we are going to prove Theorem 1.5. Throughout the section, we will assume that the hypotheses of Theorem 1.5 hold. For each irreducible representation $\omega$ of $G$, we choose an irreducible representation $\rho_{\omega}$ of $\Gamma$ such that $\left[\rho_{\omega}\right]=\phi([\omega])$.

Lemma 3.1. (a) Let $\omega^{\prime}$ and $\omega^{\prime \prime}$ be two irreducible representations of $G$, and let $\omega^{\prime} \otimes \omega^{\prime \prime} \cong \bigoplus \omega_{i}$ be a decomposition of their tensor product into irreducibles. Then $\rho_{\omega^{\prime}} \otimes \rho_{\omega^{\prime \prime}} \cong \bigoplus \rho_{\omega_{i}}$.

(b) If $\omega$ is a trivial (one-dimensional) representation 1 of $G$, then $\rho_{\omega}$ is a trivial representation of $\Gamma$.

(c) The representation $\omega$ is one-dimensional if and only if $\rho_{\omega}$ is one-dimensional.

(d) For each irreducible representation $\omega$ of $G$, we have $\rho_{\omega^{*}} \cong\left(\rho_{\omega}\right)^{*}$. 
(e) Let $\omega^{\prime}$ and $\omega^{\prime \prime}$ be two irreducible representations of $G$ such that $\rho_{\omega^{\prime}} \cong \rho_{\omega^{\prime \prime}}$. Then restrictions $\left.\omega^{\prime}\right|_{G^{\mathrm{der}}}$ and $\left.\omega^{\prime \prime}\right|_{G^{\mathrm{der}}}$ are isomorphic.

Proof. (a) By hypothesis, we have $\left[\omega^{\prime}\right] \cdot\left[\omega^{\prime \prime}\right]=\sum_{i}\left[\omega_{i}\right]$. Since $\phi$ is a homomorphism of semirings, we conclude that

$$
\left[\rho_{\omega^{\prime}} \otimes \rho_{\omega^{\prime \prime}}\right]=\phi\left(\left[\omega^{\prime}\right]\right) \cdot \phi\left(\left[\omega^{\prime \prime}\right]\right)=\sum_{i} \phi\left(\left[\omega_{i}\right]\right)=\left[\bigoplus \rho_{\omega_{i}}\right]
$$

Since $\rho_{\omega^{\prime}}$ and $\rho_{\omega^{\prime \prime}}$ are irreducible, their tensor product $\rho_{\omega^{\prime}} \otimes \rho_{\omega^{\prime \prime}}$ is semisimple (see [Chevalley 1955, p. 88]). Therefore, $\rho_{\omega^{\prime}} \otimes \rho_{\omega^{\prime \prime}} \cong \bigoplus \rho_{\omega_{i}}$.

(b) This follows from the observation that $\omega=\mathbf{1}$ if and only if $\omega \otimes \omega \cong \omega$.

(c) This follows from the observation that $\omega$ is one-dimensional if and only if $\omega \otimes \omega$ is irreducible.

(d) Note that the representation $\omega \otimes \omega^{*}$ has a trivial subrepresentation 1. Therefore, by (a) and (b), the representation $\rho_{\omega^{*}} \otimes \rho_{\omega}$ has a subrepresentation $\rho_{\mathbf{1}} \cong \mathbf{1}$. Since $\rho_{\omega}$ and $\rho_{\omega^{*}}$ are irreducible, this implies that $\rho_{\omega^{*}} \cong\left(\rho_{\omega}\right)^{*}$.

(e) If $\rho_{\omega^{\prime}} \cong \rho_{\omega^{\prime \prime}}$, then the tensor product $\rho_{\omega^{\prime}} \otimes\left(\rho_{\omega^{\prime \prime}}\right)^{*} \cong \rho_{\omega^{\prime}} \otimes \rho_{\omega^{\prime \prime *}}$ contains a subrepresentation 1. Using (a) and (c), we conclude that the tensor product $\omega^{\prime} \otimes \omega^{\prime \prime}$ * has a one-dimensional subrepresentation $\xi$. Since $\omega^{\prime}$ and $\omega^{\prime \prime}$ are irreducible, we conclude that $\omega^{\prime} \cong \omega^{\prime \prime} \otimes \xi$; thus, the restrictions $\left.\omega^{\prime}\right|_{G^{\text {der }}}$ and $\left.\omega^{\prime \prime}\right|_{G^{\text {der }}}$ are isomorphic.

3.2. For every irreducible representation $\omega$ of $G$, we denote by $z_{\omega}$ its central character. Let $Z$ be the center of $G$, and denote by $\iota$ the embedding $\Gamma \stackrel{\sim}{\longrightarrow} \Gamma \times\{1\} \hookrightarrow$ $\Gamma \times Z(K)$.

Lemma 3.3. (a) There exists a unique homomorphism of semirings $\tilde{\phi}: K_{0}^{+}[G] \rightarrow$ $K_{0}^{+}[\Gamma \times Z(K)]$ such that

$$
\tilde{\phi}([\omega])=\left[\rho_{\omega} \otimes z_{\omega}\right] \quad \text { for each irreducible } \omega .
$$

(b) The map $\tilde{\phi}$ is injective, maps irreducibles to irreducibles, and satisfies $\iota^{*} \circ \tilde{\phi}=\phi$.

(c) Assume that there exists a homomorphism $\rho: \Gamma \rightarrow G(K)$ such that $\rho^{*}=\phi$, and let $\tilde{\rho}: \Gamma \times Z(K) \rightarrow G(K)$ be a homomorphism defined by $\tilde{\rho}(\gamma, z):=\rho(\gamma) \cdot z$. Then $\tilde{\rho}^{*}=\tilde{\phi}$.

Proof. (a) Since the additive Grothendieck semigroup $K_{0}^{+}[G]$ is freely generated by irreducible elements $[\omega]$, there exists a unique homomorphism of semigroups $\tilde{\phi}: K_{0}^{+}[G] \rightarrow K_{0}^{+}[\Gamma \times Z(K)]$ that satisfies (3-1). It remains to show that for every two representations $\omega^{\prime}$ and $\omega^{\prime \prime}$ of $G$ we have an equality

$$
\tilde{\phi}\left(\left[\omega^{\prime}\right] \cdot\left[\omega^{\prime \prime}\right]\right)=\tilde{\phi}\left(\left[\omega^{\prime}\right]\right) \cdot \tilde{\phi}\left(\left[\omega^{\prime \prime}\right]\right) .
$$

By the additivity of $\tilde{\phi}$, we may assume that $\omega^{\prime}$ and $\omega^{\prime \prime}$ are irreducible. Let $\omega^{\prime} \otimes \omega^{\prime \prime} \cong \bigoplus \omega_{i}$ be a decomposition of their tensor product into irreducibles. Then 
$\left[\omega^{\prime}\right] \cdot\left[\omega^{\prime \prime}\right]=\sum_{i}\left[\omega_{i}\right]$; hence, the left-hand side of (3-2) is equal to

$$
\tilde{\phi}\left(\sum_{i}\left[\omega_{i}\right]\right)=\sum_{i} \tilde{\phi}\left(\left[\omega_{i}\right]\right)=\sum_{i}\left[\rho_{\omega_{i}} \otimes z_{\omega_{i}}\right]
$$

while the right-hand side of (3-2) is equal to

$$
\left[\rho_{\omega^{\prime}} \otimes z_{\omega^{\prime}}\right] \cdot\left[\rho_{\omega^{\prime \prime}} \otimes z_{\omega^{\prime \prime}}\right]=\left[\left(\rho_{\omega} \otimes \rho_{\omega^{\prime \prime}}\right) \otimes z_{\omega^{\prime}} z_{\omega^{\prime \prime}}\right] .
$$

Since the central character of each $\omega_{i}$ is equal to $z_{\omega^{\prime}} z_{\omega^{\prime \prime}}$, equality (3-2) follows from Lemma 3.1(a).

(b) By construction, for each irreducible element $[\omega]$, the element $\tilde{\phi}([\omega])=\left[\rho_{\omega} \otimes z_{\omega}\right]$ is irreducible, and

$$
\iota^{*} \tilde{\phi}([\omega])=\iota^{*}\left(\left[\rho_{\omega} \otimes z_{\omega}\right]\right)=\left[\rho_{\omega}\right]=\phi([\omega]) .
$$

This implies that $\tilde{\phi}$ maps irreducibles to irreducibles and satisfies $\iota^{*} \circ \tilde{\phi}=\phi$.

Finally, since as additive semigroups $K_{0}^{+}[G]$ and $K_{0}^{+}[\Gamma]$ are freely generated by irreducibles, in order to show that $\tilde{\phi}$ is injective, it is enough to show that it is injective on irreducibles.

Let $\omega^{\prime}$ and $\omega^{\prime \prime}$ be two irreducible representations of $G$ such that $\tilde{\phi}\left(\left[\omega^{\prime}\right]\right)=\tilde{\phi}\left(\left[\omega^{\prime \prime}\right]\right)$. Then $\rho_{\omega^{\prime}} \cong \rho_{\omega^{\prime \prime}}$ and $z_{\omega^{\prime}}=z_{\omega^{\prime \prime}}$. Using Lemma 3.1(e), we conclude that $\left.\omega^{\prime}\right|_{G^{\text {der }}} \cong$ $\left.\omega^{\prime \prime}\right|_{G^{\text {der }}}$ and $\left.\omega^{\prime}\right|_{Z}=\left.\omega^{\prime \prime}\right|_{Z}$. Hence, $\omega^{\prime} \cong \omega^{\prime \prime}$, implying the injectivity.

(c) It is enough to show that $\tilde{\rho}^{*}([\omega])=\tilde{\phi}([\omega])$ when $[\omega]$ is irreducible. Both expressions, however, are equal to $\left[\rho_{\omega} \otimes z_{\omega}\right]$.

3.4. Proof of Theorem 1.5. First we will show the existence of $\rho$ under the assumption that $\phi: K_{0}^{+}[G] \rightarrow K_{0}^{+}[\Gamma]$ is injective.

Let $\mathscr{C}$ be the full subcategory of Rep $\Gamma$ consisting of semisimple representations $\tau \in \operatorname{Rep} \Gamma$ such that $[\tau]=\phi([\omega])$ for some $[\omega] \in K_{0}^{+}[G]$. Since $\phi([\omega])$ is irreducible for each irreducible $[\omega], \mathscr{C}$ is a semisimple abelian subcategory. Since $\phi$ is a homomorphism of semirings, $\mathscr{C}$ is a rigid tensor subcategory of Rep $\Gamma$ (use Lemma 3.1(a)-(d)) and hence a Tannakian category. Let $f: \mathscr{C} \rightarrow \operatorname{Vec}_{\mathrm{K}}$ be the forgetful functor, and let $H:=\operatorname{Aut}^{\otimes}(f)$ be the group of tensor automorphisms of $f$.

By the Tannakian formalism (see, for example, [Deligne and Milne 1982, Theorem 2.11]), $H$ is an affine group scheme, and $f$ induces an equivalence of tensor categories $\mathscr{C} \stackrel{\sim}{\longrightarrow} \operatorname{Rep} H$. Since $G$ is an algebraic group, the category $\operatorname{Rep} G$ has a tensor generator $\omega$. Then an element $\rho_{\omega} \in \operatorname{Rep} \Gamma$ such that $\left[\rho_{\omega}\right]=\phi([\omega])$ must be a tensor generator of $\mathscr{C} \cong \operatorname{Rep} H$. This implies that $H$ is an algebraic group (see [Deligne and Milne 1982, Proposition 2.20]). Moreover, since $\mathscr{C} \cong \operatorname{Rep} H$ is semisimple, the group $H$ is reductive (see [Deligne and Milne 1982, Proposition 2.23]).

Every element of $\gamma \in \Gamma$ defines a tensor automorphism of $f$ over $K$. Hence we get a group homomorphism $\pi: \Gamma \rightarrow H(K)$ such that $\pi^{*}: \operatorname{Rep} H \rightarrow \operatorname{Rep} \Gamma$ is the inverse of the equivalence $f: \mathscr{C} \stackrel{\sim}{\longrightarrow} \operatorname{Rep} H$. 
By construction, the homomorphism $\phi: K_{0}^{+}[G] \rightarrow K_{0}^{+}[\Gamma]$ decomposes as $K_{0}^{+}[G] \stackrel{\phi^{\prime}}{\rightarrow} K_{0}^{+}[H] \stackrel{\pi^{*}}{\rightarrow} K_{0}^{+}[\Gamma]$, and the homomorphism $\phi^{\prime}$ is surjective. By our assumption, $\phi^{\prime}$ is also injective; hence, it is an isomorphism. Since $G$ is connected, we conclude that $H$ is connected as well (use, for example, [Deligne and Milne 1982, Corollary 2.22]). Therefore, by Theorem 1.2, there exists an isomorphism $\rho^{\prime}: H \stackrel{\sim}{\rightarrow} G$ such that $\phi^{\prime}=\rho^{\prime *}$. Then the composition $\rho:=\rho^{\prime} \circ \pi: \Gamma \rightarrow G(K)$ satisfies $\rho^{*}=\pi^{*} \circ \phi^{\prime}=\phi$.

To show the existence of $\rho$ in general, we consider the homomorphism of Grothendieck semirings $\tilde{\phi}: K_{0}^{+}[G] \rightarrow K_{0}^{+}[\Gamma \times Z(K)]$, considered in Lemma 3.3(a).

Then $\tilde{\phi}$ is injective, so by the particular case shown above, there exists a homomorphism $\tilde{\rho}: \Gamma \times Z(K) \rightarrow G(K)$ such that $\tilde{\rho}^{*}=\tilde{\phi}$. Then the composition $\rho:=\tilde{\rho} \circ \iota: \Gamma \rightarrow G(K)$ satisfies $\rho^{*}=\iota^{*} \circ \tilde{\phi}=\phi$.

Conversely, let $\rho: \Gamma \rightarrow G(K)$ be a homomorphism such that $\rho^{*}=\phi$. To show that the Zariski closure of $\rho(\Gamma)$ contains $G^{\text {der }}$, it suffices to show that the homomorphism $\tilde{\rho}: \Gamma \times Z(K) \rightarrow G(K)$ from Lemma 3.3(c) has a Zariski closed image.

Let $H \subset G$ be the Zariski closure of the image of $\tilde{\rho}$, and denote by $i$ the inclusion $H \hookrightarrow G$. Then $\tilde{\rho}^{*}=\tilde{\phi}: K_{0}^{+}[G] \rightarrow K_{0}^{+}[\Gamma \times Z(K)]$ factors through $i^{*}: K_{0}^{+}[G] \rightarrow K_{0}^{+}[H]$. In particular, $i^{*}$ is injective and maps irreducibles to irreducibles. Then using Chevalley's theorem ([Borel 1991, Theorem 5.1] or [Deligne and Milne 1982, Proposition 2.21]), $i$ has to be an isomorphism.

Finally, to show that $\rho$ is unique up to conjugation, it suffice to show that $\tilde{\rho}: \Gamma \times Z(K) \rightarrow G(K)$ is unique up to conjugation. Thus, we can replace $\rho$ by $\tilde{\rho}$ and $\phi$ by $\tilde{\phi}$, thereby assuming that $\phi$ is injective.

Then, using the notation of the existence part, the tensor functor $\rho^{*}: \operatorname{Rep} G \rightarrow$ $\operatorname{Rep} \Gamma$ decomposes as a composition $\operatorname{Rep} G \stackrel{\psi}{\rightarrow} \operatorname{Rep} H \stackrel{\pi^{*}}{\rightarrow} \operatorname{Rep} \Gamma$ of tensor functors. By the Tannakian formalism, there exists a homomorphism $\rho^{\prime}: H \rightarrow G$ such that $\rho^{\prime *}=\psi$. Then $\rho$ is conjugate to the composition $\rho^{\prime} \circ \pi$, so it remains to show that the conjugacy class of $\rho^{\prime}$ is uniquely defined.

We have seen that $\phi$ decomposes as $K_{0}^{+}[G] \stackrel{\phi^{\prime}}{\rightarrow} K_{0}^{+}[H] \stackrel{\pi^{*}}{\rightarrow} K_{0}^{+}[\Gamma]$; therefore, $\rho^{\prime *}: K_{0}^{+}[G] \stackrel{\sim}{\rightarrow} K_{0}^{+}[H]$ coincides with $\phi^{\prime}$. Hence, the uniqueness assertion for $\rho^{\prime}$ follows from Theorem 1.2.

\section{Two corollaries}

In this section, we are going to prove Corollaries 1.8 and 1.11.

Lemma 4.1. Assume that the hypotheses of Corollary 1.8 hold.

(a) There exists a unique homomorphism of semirings $\phi: K_{0}^{+}[G] \rightarrow K_{0}^{+}[\Gamma]$ such that $\phi([\omega])=\left[\rho_{\omega}\right]$ for each irreducible $\omega$.

(b) Let $\rho: \Gamma \rightarrow G(K)$ be a group homomorphism. Then equality (1-2) holds for $\rho$ if and only if $\omega \circ \rho \cong \rho_{\omega}$ for all irreducible $\omega$. 
Proof. (a) Since the semigroup $K_{0}^{+}[G]$ is freely generated by irreducible elements, there exists a unique homomorphism of semigroups $\phi: K_{0}^{+}[G] \rightarrow K_{0}^{+}[\Gamma]$ such that $\phi([\omega])=\left[\rho_{\omega}\right]$ for each irreducible $\omega$.

It remains to show that for every two representations $\omega^{\prime}$ and $\omega^{\prime \prime}$ of $G$ we have an equality

$$
\phi\left(\left[\omega^{\prime}\right] \cdot\left[\omega^{\prime \prime}\right]\right)=\phi\left(\left[\omega^{\prime}\right]\right) \cdot \phi\left(\left[\omega^{\prime \prime}\right]\right)
$$

Since a semisimple representation is determined by its trace, it is enough to show that

$$
\operatorname{Tr}_{\phi\left(\left[\omega^{\prime}\right] \cdot\left[\omega^{\prime \prime}\right]\right)}(\gamma)=\operatorname{Tr}_{\phi\left(\left[\omega^{\prime}\right]\right)}(\gamma) \cdot \operatorname{Tr}_{\phi\left(\left[\omega^{\prime \prime}\right]\right)}(\gamma)
$$

for all $\gamma \in \Gamma$. First we observe that for all $\gamma \in \Gamma$ and all $[\omega] \in K_{0}^{+}[G]$ we have an equality

$$
\operatorname{Tr}_{\phi([\omega])}(\gamma)=\operatorname{Tr}_{[\omega]}(f(\gamma)) .
$$

Indeed, by additivity, it is enough to show (4-2) for $\omega$ irreducible. In this case, the assertion follows from equalities $\phi([\omega])=\left[\rho_{\omega}\right]$ and $(1-1)$.

Using (4-2), our desired equality (4-1) can be written in the form

$$
\operatorname{Tr}_{\left[\omega^{\prime}\right] \cdot\left[\omega^{\prime \prime}\right]}(f(\gamma))=\operatorname{Tr}_{\left[\omega^{\prime}\right]}(f(\gamma)) \cdot \operatorname{Tr}_{\left[\omega^{\prime \prime}\right]}(f(\gamma)) .
$$

Therefore, it follows from the multiplicativity of the trace map $\operatorname{Tr}: K_{0}^{+}[G] \rightarrow K[G]$. (b) Since functions $\operatorname{Tr}_{\omega}$ with $\omega$ irreducible generate $K\left[c_{G}\right]$ as a $K$-vector space (see [Steinberg 1965, Theorem 6.1(a)]), the equality (1-2) is equivalent to the equality

$$
\operatorname{Tr}_{\omega}\left(\chi_{G}(\rho(\gamma))\right)=\operatorname{Tr}_{\omega}(f(\gamma))
$$

for all $\gamma \in \Gamma$ and all irreducible $\omega$. Since the left side of (4-3) equals $\operatorname{Tr}_{\omega \circ \rho}(\gamma)$ (see 1.7(b)) while the right-hand side of (4-3) equals $\operatorname{Tr}_{\rho_{\omega}}(\gamma)$ by (1-1), equality (4-3) is equivalent to the equality $\operatorname{Tr}_{\omega \circ \rho}=\operatorname{Tr}_{\rho_{\omega}}$ for all irreducible $\omega$. But this is equivalent to the desired isomorphism $\omega \circ \rho \cong \rho_{\omega}$.

4.2. Proof of Corollary 1.8. By Lemma 4.1(a), there exists a unique homomorphism of semirings $\phi: K_{0}^{+}[G] \rightarrow K_{0}^{+}[\Gamma]$ such that $\phi([\omega])=\left[\rho_{\omega}\right]$ for each irreducible $\omega$. Then by Theorem 1.5, there exists a homomorphism $\rho: \Gamma \rightarrow G(K)$ such that $\rho^{*}=\phi$. In particular, we have that $[\omega \circ \rho]$ is equal to $\phi([\omega])=\left[\rho_{\omega}\right]$ for each irreducible $\omega$. Then by Lemma 4.1(b), the equality (1-2) holds for $\rho$.

Conversely, let $\rho: \Gamma \rightarrow G(K)$ be a homomorphism, satisfying (1-2). Then by Lemma 4.1(b), $\rho^{*}([\omega])=[\omega \circ \rho]$ is equal to $\phi([\omega])=\left[\rho_{\omega}\right]$ for each irreducible $\omega$. Thus $\rho^{*}: K_{0}^{+}[G] \rightarrow K_{0}^{+}[\Gamma]$ is equal to $\phi$. It then follows from Theorem 1.5 that $\rho$ is unique up to conjugation, and that the Zariski closure of $\rho(\Gamma)$ contains $G^{\mathrm{der}}$.

4.3. Proof of Corollary 1.11. The argument is very similar to that of Corollary 1.8. 
As in Lemma 4.1(a), there exists a unique homomorphism of semirings

$$
\phi: K_{0}^{+}[\hat{G}] \rightarrow K_{0}^{+}\left[\Gamma_{F}\right]
$$

such that $\phi([\omega])=\left[\rho_{\omega}\right]$ for each irreducible $\omega$. Indeed, arguing as in Lemma 4.1(a) word for word, we reduce ourselves to the equality (4-1). Moreover, by the Chebotarev density theorem, it is enough to show equality (4-1) when $\gamma=$ Frob $_{v}$ for almost all $v \in \Sigma$.

Then we reduce the problem to showing that

$$
\operatorname{Tr}_{\phi([\omega])}\left(\operatorname{Frob}_{v}\right)=\operatorname{Tr}_{[\omega]}(f(v))
$$

for all irreducible $[\omega]$ and almost all $v \in \Sigma$. But the latter equality follows from equalities $\phi([\omega])=\left[\rho_{\omega}\right]$ and (1-3).

By Theorem 1.5, there now exists a homomorphism $\rho: \Gamma_{F} \rightarrow \hat{G}\left(\overline{\mathbb{Q}}_{\ell}\right)$ such that $\rho^{*}=\phi$.

We claim that, for every representation $\omega$ of $\hat{G}$, the composition $\omega \circ \rho$ is a semisimple $\ell$-adic representation. By additivity, it is enough to show in the case when $\omega$ is irreducible. However, in this case,

$$
[\omega \circ \rho]=\rho^{*}([\omega])=\left[\rho_{\omega}\right]
$$

is irreducible; hence, $\omega \circ \rho \cong \rho_{\omega}$ is an irreducible $\ell$-adic representation.

Choosing $\omega$ to be a faithful representation of $\hat{G}$, we conclude that $\rho$ is continuous and unramified almost everywhere.

Finally, arguing exactly as in Lemma 4.1(b) (and using the isomorphisms $\left.\omega \circ \rho \cong \rho_{\omega}\right)$, we conclude that $\rho$ satisfies the equality (1-4).

Conversely, let $\rho: \Gamma_{F} \rightarrow \hat{G}\left(\overline{\mathbb{Q}}_{\ell}\right)$ be a $\hat{G}$-valued $\ell$-adic representation satisfying (1-4). Again arguing exactly as in Lemma 4.1(b) and using the Chebotarev density theorem, we conclude that $\rho^{*}([\omega])=[\omega \circ \rho]$ is equal to $\phi([\omega])=\left[\rho_{\omega}\right]$ for each irreducible $\omega$. Thus, $\rho^{*}: K_{0}^{+}[\hat{G}] \rightarrow K_{0}^{+}\left[\Gamma_{F}\right]$ is equal to $\phi$.

Therefore, it follows from Theorem 1.5 that $\rho$ is unique up to conjugation and that the Zariski closure of $\rho\left(\Gamma_{F}\right)$ contains $\hat{G}^{\text {der }}$.

\section{Acknowledgments}

We would like to acknowledge the contribution of Pavel Etingof, whose suggestions led to substantial simplification and conceptual clarification of this paper.

Part of the work was done while Kazhdan and Varshavsky visited the University of Chicago. The rest of the work was done while Varshavsky visited Indiana University. We thank both these institutions for stimulating atmosphere and financial support. We would also like to thank Michael Mueger for calling our attention to two previous proofs of Theorem 1.2. 


\section{References}

[Borel 1991] A. Borel, Linear algebraic groups, 2nd ed., Graduate Texts in Mathematics 126, Springer, New York, 1991. MR 92d:20001 Zbl 0726.20030

[Chevalley 1955] C. Chevalley, Théorie des groupes de Lie, III: Théorèmes généraux sur les algèbres de Lie, Actualités Sci. Ind. 1226, Hermann \& Cie, Paris, 1955. MR 16,901a

[Deligne and Milne 1982] P. Deligne and J. S. Milne, "Tannakian categories", pp. 101-228 in Hodge cycles, motives, and Shimura varieties, Lecture Notes in Mathematics 900, Springer, Berlin, 1982. MR 84m:14046 Zbl 0477.14004

[Handelman 1993] D. Handelman, "Representation rings as invariants for compact groups and limit ratio theorems for them”, Internat. J. Math. 4:1 (1993), 59-88. MR 94c:22005 Zbl 0788.22005

[Kazhdan and Varshavsky $\geq 2014$ ] D. Kazhdan and Y. Varshavsky, "On the cohomology of the moduli spaces of $F$-bundles: stable cuspidal Deligne-Lusztig part", In preparation.

[Kumar 1988] S. Kumar, "Proof of the Parthasarathy-Ranga Rao-Varadarajan conjecture", Invent. Math. 93:1 (1988), 117-130. MR 89j:17009 Zbl 0668.17008

[Larsen and Pink 1990] M. Larsen and R. Pink, "Determining representations from invariant dimensions", Invent. Math. 102:2 (1990), 377-398. MR 92c:22026 Zbl 0687.22004

[McMullen 1984] J. R. McMullen, "On the dual object of a compact connected group", Math. Z. 185:4 (1984), 539-552. MR 85e:22010 Zbl 0513.43007

[Steinberg 1965] R. Steinberg, "Regular elements of semisimple algebraic groups", Inst. Hautes Études Sci. Publ. Math. 25 (1965), 49-80. MR 31 \#4788 Zbl 0136.30002

Communicated by Richard Taylor

Received 2012-09-02 Revised 2013-08-20 Accepted 2013-09-19

kazhdan@math.huji.ac.il Einstein Institute of Mathematics, Hebrew University, Givat Ram, 91904 Jerusalem, Israel

mjlarsen@indiana.edu

Department of Mathematics, Indiana University, Rawles Hall, Bloomington, IN 47405-5701, United States

vyakov@math.huji.ac.il

Einstein Institute of Mathematics, Hebrew University, Givat Ram, 91904 Jerusalem, Israel 


\section{Algebra \& Number Theory}

msp.org/ant

\section{EDITORS}

MANAGING EDITOR

Bjorn Poonen

Massachusetts Institute of Technology

Cambridge, USA

\author{
EDITORIAL BOARD CHAIR \\ David Eisenbud \\ University of California \\ Berkeley, USA
}

\section{BOARD OF EDITORS}

Georgia Benkart

Dave Benson

Richard E. Borcherds

John H. Coates

J-L. Colliot-Thélène

Brian D. Conrad

Hélène Esnault

Hubert Flenner

Edward Frenkel

Andrew Granville

Joseph Gubeladze

Roger Heath-Brown

Ehud Hrushovski

Craig Huneke

Mikhail Kapranov

Yujiro Kawamata

János Kollár

Yuri Manin

Barry Mazur

Philippe Michel
University of Wisconsin, Madison, USA

University of Aberdeen, Scotland

University of California, Berkeley, USA

University of Cambridge, UK

CNRS, Université Paris-Sud, France

University of Michigan, USA

Freie Universität Berlin, Germany

Ruhr-Universität, Germany

University of California, Berkeley, USA

Université de Montréal, Canada

San Francisco State University, USA

Oxford University, UK

Hebrew University, Israel

University of Virginia, USA

Yale University, USA

University of Tokyo, Japan

Princeton University, USA

Northwestern University, USA

Harvard University, USA

École Polytechnique Fédérale de Lausanne
Susan Montgomery

Shigefumi Mori

Raman Parimala

Jonathan Pila

Victor Reiner

Karl Rubin

Peter Sarnak

Joseph H. Silverman

Michael Singer

Vasudevan Srinivas

J. Toby Stafford

Bernd Sturmfels

Richard Taylor

Ravi Vakil

Michel van den Bergh

Marie-France Vignéras

Kei-Ichi Watanabe

Efim Zelmanov

Shou-Wu Zhang
University of Southern California, USA

RIMS, Kyoto University, Japan

Emory University, USA

University of Oxford, UK

University of Minnesota, USA

University of California, Irvine, USA

Princeton University, USA

Brown University, USA

North Carolina State University, USA

Tata Inst. of Fund. Research, India

University of Michigan, USA

University of California, Berkeley, USA

Harvard University, USA

Stanford University, USA

Hasselt University, Belgium

Université Paris VII, France

Nihon University, Japan

University of California, San Diego, USA

Princeton University, USA

PRODUCTION

production@msp.org

Silvio Levy, Scientific Editor

See inside back cover or msp.org/ant for submission instructions.

The subscription price for 2014 is US $\$ 225 /$ year for the electronic version, and $\$ 400 /$ year $(+\$ 55$, if shipping outside the US) for print and electronic. Subscriptions, requests for back issues and changes of subscribers address should be sent to MSP.

Algebra \& Number Theory (ISSN 1944-7833 electronic, 1937-0652 printed) at Mathematical Sciences Publishers, 798 Evans Hall \#3840, c/o University of California, Berkeley, CA 94720-3840 is published continuously online. Periodical rate postage paid at Berkeley, CA 94704, and additional mailing offices.

ANT peer review and production are managed by EditFLOW ${ }^{\circledR}$ from Mathematical Sciences Publishers.

\section{PUBLISHED BY}

- mathematical sciences publishers

nonprofit scientific publishing

http://msp.org/

(C) 2014 Mathematical Sciences Publishers 


\section{Algebra \& Number Theory}

\section{Volume $8 \quad$ No. $1 \quad 2014$}

On the Picard number of K3 surfaces over number fields

FRANÇOIS CHARLES

Adèle residue symbol and Tate's central extension for multiloop Lie algebras

OLIVER BRAUNLING

On the number of cubic orders of bounded discriminant having automorphism group $C_{3}$, and related problems

MANJUl Bhargava and ARIEl ShNidman

Polynomial bounds for Arakelov invariants of Belyi curves

ARIYAN JAVANPEYKAR

The radius of a subcategory of modules

HAILONG DAO and RYO TAKAHASHI

A generalized Bogomolov-Gieseker inequality for the three-dimensional projective space

EMANUELE MACRÌ

$(\varphi, \Gamma)$-modules over noncommutative overconvergent and Robba rings

GERGELY ZÁBRÁDI

The Tannakian formalism and the Langlands conjectures

DAVID KAZHDAN, MICHAEL LARSEN and YAKOV VARSHAVSKY 